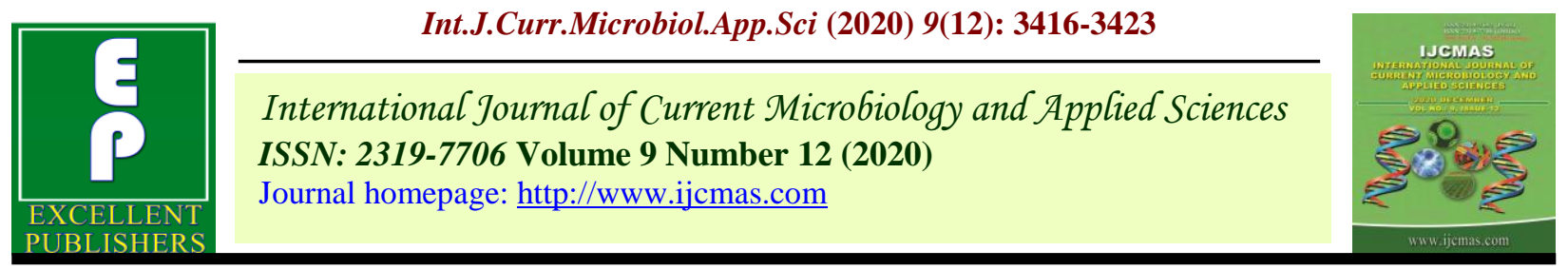

Original Research Article

https://doi.org/10.20546/ijcmas.2020.912.406

\title{
Genetic Variability, Heritability and Genetic Advance Studies on Rabi Maize (Zea mays L.)
}

\author{
Abhishek Sadhu*, Amitava Paul, P. C. Kole and Sandip Debnath \\ Department of Genetics and Plant Breeding, Palli Siksha Bhavana (Institute of Agriculture), \\ Visva Bharati, Bolpur, West Bengal, India \\ *Corresponding author
}

\section{A B S T R A C T}

\begin{tabular}{|c|}
\hline Keywords \\
\hline $\begin{array}{l}\text { Maize genotypes, } \\
\text { Genetic variability, } \\
\text { Heritability, } \\
\text { Genetic advance, } \\
\text { GCV, PCV }\end{array}$ \\
\hline Article Info \\
\hline $\begin{array}{l}\text { Accepted: } \\
28 \text { November } 2020 \\
\text { Available Online: } \\
10 \text { December } 2020\end{array}$ \\
\hline
\end{tabular}

Keywords

Maize genotypes, Genetic variability, Heritability, Genetic advance,

\section{Introduction}

Maize (Zea mays L.) $(2 \mathrm{n}=2 \mathrm{x}=20)$ is an important cereal crop belonging to the Poaceae family and tribe Maydeae. Centre of origin of maize is believed to be in Southern Mexico and had been domesticated about 7000 years ago. Because of its high genetic yield potential compared to other cereal crops it is also known as "queen of cereals". Maize exhibits greater diversity in phenotype and habitat than any other cereal crop. It can be cultivated from tropical to temperate regions of the world. It is cultivated over a wide range of climatic conditions, from latitude $58^{0} \mathrm{~N}$ to $40^{0} \mathrm{~S}$, from mean sea level to altitude above $3000 \mathrm{~m}$ and in areas receiving annual rainfall from $250 \mathrm{~mm}$ to $5000 \mathrm{~mm}$, making it considered as a promising option for diversifying agriculture in various agroclimatic zones. It is grown at 8.67 mha with $22.25 \mathrm{mt}$ of production and an average productivity of $2.5-3 \mathrm{t} \mathrm{ha}^{-\mathrm{I}}$ (Economics and Statistics Department, 2018). At present, maize accounts for nearly $9 \%$ of the national food basket and more than 400 billion rupees of GDP of our country. Among other cereals maize is rich in starch, sucrose and oil 
content. Globally 67 percent of maize is used for livestock feed, $25 \%$ human consumption, industrial purposes and balance is used as seed and demand for grain is increasing worldwide.

Making all these aspects in consideration, plant breeder is particularly interested in genetic diversity of maize as it plays a crucial role in performing successful breeding program. The heritability of a metric trait is a parameter of particular importance to the breeder, since it tests the degree of resemblance between the parents and the offspring and its magnitude indicates the heritability with which a genotype can be defined by its phenotypic expression while genetic advance aids in exercising the necessary selection intensity. Studying variations, heritability and genetic advance in the genotypes can help assess the true potential value of the genotypes. Heritability and genetic advance are very important parameters for selection of genotypes; however, it is not necessary that characters showing high heritability will be definitely show high genetic advance. Heritability is a heritable portion of a phenotypic variance in which the estimation of heritability helps the plant breeder to selecting out elite genotype from diverse genetic population. Genetic advance is a measure of genetic gain under the selection and understanding types of gene action for polygenic traits.

\section{Materials and Methods}

The field experiment was conducted in one season at the Agricultural Farm of Palli Siksha Bhavana (Institute of Agriculture), Visva-Bharati, Sriniketan. The experimental plant material in the present investigation comprised of forty genotypic lines of maize obtained from Indian Institute of Maize Research (IIMR), Winter Nursery Centre (WNC), Hyderabad. The experiment was carried out during rabi 2018. The design adopted for this experiment was Randomized Block Deign (RBD) with 3 replications. Each entry in each replication was sown in 5 rows of $2.5 \mathrm{~m}$ length with spacing $60 \mathrm{~cm}$ apart from each other with plant to plant distance of $20 \mathrm{~cm}$. The recommended doses of fertilizers such as $\mathrm{N}, \mathrm{P}$ and $\mathrm{K}$ were applied in the ratio of 120:60:60 kg ha ${ }^{-1}$. The entire $P$ and $K$ and one third dose of nitrogen was applied as basal dose while remaining dose of $\mathrm{N}$ was applied in two equal split doses at knee height and tasseling stages. Irrigation and intercultural operations like weeding were followed as per shedules. Necessary prophylactic plant protection measures were adopted to protect the crop from pests and diseases as per the guidelines of IIMR recommendations, so as to raise a normal healthy crop. Observations on fifteen different quantitative characters were recorded on ten randomly selected competitive plants for each genotype in various phenotypes of the crop except for day's to $50 \%$ tasseling, days to $50 \%$ silking and days to maturity where data is recorded on plot basis. Mean values from the ten randomly selected plants from each of the three replications were averaged and expressed as the mean of the respective character. The mean values were considered for statistical analysis of variance (ANOVA) was done as per the procedure given by (Panse, 1957) following the procedure of Randomized Block Design (RBD) analysis, estimation of broad sense heritability was calculated by formula given by to Hanson et al.,(1956), Genetic advance in per cent of mean was estimated by the formula given by Comstock and Robinson et al., (1949).

\section{Results and Discussion}

Genetic variability: The mean performance valus for 40 maize genotypes for 15 quantitative characters and analysis of variance for the same are given in table $1 \& 2$ 
indicated that plant height, cobs per plant, ear height, ear leaf length, shelling (\%), test weight, cob wt, no. of kernel per row, days $75 \%$ dry husk, exhibited significant difference among 40 maize genotypes. Thus, the presence of variability among these genotypes in the present study indicated ample scope for improvement through selection of these traits. Similar results were recorded by Saleem et al., (2007), Nzuve et al., (2014), and Sharma et al., (2014).

PCV and GCV: In general the value of the phenotypic coefficient of variation (PCV) was higher than that of genotypic coefficient of variation for all the characters given in table 3 . The phenotypic coefficient of variation was estimated to be high $(>20 \%)$ for grain yield (32.37\%), cobs per plant $(23.97 \%)$, cob wt $(21.80 \%)$, ear height $(20.21 \%)$, while moderate $(20-10 \%)$ for test weight $(14.08 \%)$, ear leaf length (12.42\%), no. of kernel per row (11.20\%), plant height (11.17\%), and low $(<10 \%)$ for no. of rows per cob $(8.94 \%)$, shelling (\%) (7.95\%), cob length $(7.83 \%)$, cob diameter $(5.62 \%)$, days $50 \%$ pollen shed (3.72\%), days $50 \%$ silking $(2.07 \%)$, days $75 \%$ dry husk $(2.07 \%)$. This was confirmed by previous reports of Kumar, and Satyanarayana (2001), Akbar et al.,(2008). Those characters with low magnitude of genetic variability can have limited utility in selection for their improvement programe. Genotypic coefficient of variation (GCV) observed higher (>20\%) for cob wt (20.29\%), while moderate $(20-10 \%)$ for Cobs per plant $(13.9 \%)$, ear height $(13.5 \%)$, grain yield (13.15), low $(<10 \%)$ for test weight $(9.85 \%)$, plant height $(8.03 \%)$, no. of kernel per $\operatorname{row}(7.94 \%)$, no. of rows per cob $(7.8 \%)$, ear leaf length $(7.67 \%)$, cob length $(4.81 \%)$, cob diameter $(4.09 \%)$, shelling (\%) $(3.19 \%)$, days $50 \%$ pollen shed $(1.47 \%)$, days $75 \%$ dry husk $(1.22 \%)$, days $50 \%$ silking $(1.14 \%)$. The estimates of GCV and PCV values for characters shelling (\%), ear height, cobs per plant, test weight, ear leaf length, grain yield have shown higher difference which indicates the presence of grater environmental factors influencing the expression of this character. Very low difference was observed for characters like days $75 \%$ dry husk, days $50 \%$ pollen shed, day's 50\% silking, cob wt, cob diameter, cob length, no. of rows per cob indicating low sensitivity to environment and consequently greater role for genetic factors influencing the expression of these characters. Similar results were reported by Shakoor et al., (2007), Sharma et al., (2014), and Ghosh et al., (2014) in their findings.

\section{Heritability}

Heritability in broad sense were high for cob wt, and no of rows per cob, and moderate for plant height, cob diameter, no. of kernel per row, test weight, ear height, ear leaf length, cob length, days $75 \%$ dry husk, cobs per plant, days $50 \%$ silking. High heritability estimates for cob wt and no of rows per cob were also reported by Sumathi et al., (2005), Akbar et al., (2008), and satyanarayana (2001), while moderate estimates of heritability for plant height, cob diameter, no of kernel per row, test weight, cob length, days $75 \%$ dry husk, cobs per plant, days $50 \%$ silking were observed by Sumathi et al.,(2005), Akbar et al.,(2008), Pradeep Kuma, and Satyanarayana (2001), while moderate estimate of heritability for plant height, cob diameter, no. of kernel per row, test weight, cob length, days $75 \%$ dry husk, cobs per plant, days $50 \%$ silking were observed by singhal et al.,(2006), Bekele et al.,(2014), Sesay et al., (2016), Begum et al., (2016) and Ojo et al., (2006), estimates of heritability were low for characters like grain yield, shelling, days $50 \%$ pollen shed, which indicate higher influence of environment for the expression of these characters. These were supported with findings of Singhal et al., (2006) for days $50 \%$ pollen shed. 
Table.1 Mean performance of forty genotypes for fifteen quantitative characters in rabi maize

\begin{tabular}{|c|c|c|c|c|c|c|c|c|c|c|c|c|c|c|c|}
\hline & 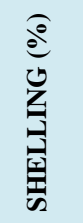 & 允总 & 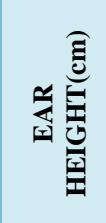 & 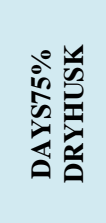 & 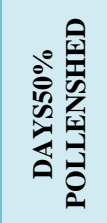 & 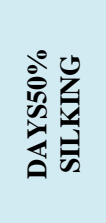 & 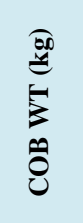 & 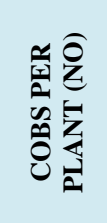 & 命䆓 & 疍 & 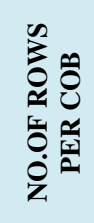 & 氞 & 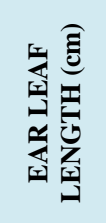 & 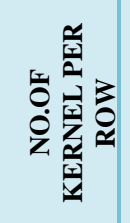 & 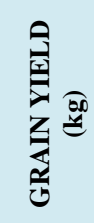 \\
\hline PM17208L & 85.18 & 280.03 & 120.93 & 134.33 & 92.00 & 100.00 & 15.43 & 125.66 & 4.27 & 27.16 & 15.40 & 30.97 & 77.10 & 27.83 & 13.51 \\
\hline ADV7043 & 85.06 & 238.53 & 95.73 & 136.66 & 95.33 & 99.66 & 14.42 & 102.66 & 4.18 & 28.30 & 13.80 & 26.52 & 91.03 & 33.13 & 13.03 \\
\hline Rasi4118 & 79.77 & 302.00 & 129.43 & 135.33 & 94.66 & 99.00 & 19.24 & 119.66 & 4.63 & 24.80 & 14.93 & 27.27 & 87.00 & 39.06 & 14.66 \\
\hline DKC9197(IS8638) & 83.62 & 287.73 & 122.23 & 137.66 & 101.66 & 100.00 & 17.42 & 97.66 & 4.30 & 25.66 & 14.66 & 30.97 & 100.20 & 36.63 & 13.80 \\
\hline PM17201L & 87.61 & 262.53 & 107.06 & 135.66 & 93.66 & 99.66 & 19.90 & 125.00 & 4.68 & 26.66 & 16.33 & 30.47 & 77.80 & 33.06 & 19.10 \\
\hline PM17205L & 86.46 & 274.50 & 97.70 & 135.33 & 94.33 & 99.00 & 16.63 & 119.66 & 4.52 & 25.31 & 15.13 & 28.04 & 77.70 & 29.60 & 14.06 \\
\hline KMH25K45(C) & 84.24 & 293.00 & 124.00 & 135.66 & 96.00 & 99.66 & 18.40 & 116.66 & 4.47 & 25.76 & 14.53 & 29.89 & 85.40 & 35.00 & 15.96 \\
\hline P3522(C) & 84.75 & 284.80 & 135.13 & 135.66 & 94.66 & 97.66 & 17.23 & 132.66 & 4.17 & 24.00 & 14.00 & 27.82 & 80.20 & 28.03 & 13.90 \\
\hline NMH713(C) & 84.69 & 243.23 & 90.80 & 135.66 & 94.33 & 99.33 & 12.04 & 95.33 & 4.41 & 24.86 & 15.53 & 27.19 & 85.36 & 30.33 & 10.30 \\
\hline GK3208 & 82.09 & 229.13 & 95.76 & 137.66 & 97.33 & 100.33 & 14.75 & 117.33 & 4.33 & 24.33 & 13.40 & 26.36 & 81.60 & 27.53 & 12.63 \\
\hline BLH 113 & 84.60 & 275.20 & 127.86 & 133.33 & 96.33 & 99.66 & 17.22 & 120.33 & 4.51 & 27.16 & 15.33 & 28.04 & 93.50 & 32.30 & 12.10 \\
\hline DKC9188 & 84.91 & 283.46 & 130.73 & 142.33 & 93.66 & 98.33 & 11.42 & 112.66 & 4.33 & 28.36 & 15.46 & 28.73 & 104.23 & 33.70 & 16.06 \\
\hline Bio305 & 79.94 & 269.73 & 122.06 & 133.66 & 97.00 & 100.00 & 17.22 & 123.00 & 4.45 & 25.76 & 15.73 & 26.45 & 100.90 & 30.26 & 14.43 \\
\hline ADV7037 & 83.54 & 227.50 & 109.56 & 138.66 & 100.00 & 100.66 & 15.54 & 128.33 & 4.10 & 28.23 & 14.53 & 23.81 & 95.06 & 31.83 & 10.57 \\
\hline DKC9181 & 85.00 & 304.50 & 122.63 & 138.33 & 97.66 & 101.00 & 18.75 & 153.00 & 4.09 & 25.50 & 12.73 & 27.66 & 97.23 & 33.40 & 13.06 \\
\hline HT16047 & 83.46 & 278.36 & 145.00 & 133.66 & 96.33 & 99.00 & 15.36 & 114.00 & 4.20 & 28.93 & 14.06 & 22.86 & 96.81 & 29.40 & 11.03 \\
\hline PM16202L & 83.38 & 297.20 & 143.30 & 132.33 & 93.33 & 98.33 & 16.00 & 154.00 & 4.31 & 29.26 & 13.73 & 28.69 & 91.00 & 29.70 & 15.80 \\
\hline PM16201L & 81.86 & 286.76 & 108.40 & 138.33 & 94.00 & 98.00 & 15.84 & 136.00 & 4.49 & 26.10 & 17.20 & 25.43 & 98.80 & 27.70 & 14.46 \\
\hline VNR32994 & 85.16 & 252.37 & 107.63 & 139.66 & 97.00 & 98.66 & 11.89 & 117.33 & 4.31 & 25.33 & 16.40 & 21.81 & 84.90 & 28.90 & 10.22 \\
\hline PM16205LPM5L & 85.70 & 265.63 & 125.60 & 133.33 & 92.33 & 96.66 & 17.28 & 146.33 & 4.17 & 25.30 & 13.26 & 29.28 & 91.06 & 26.20 & 14.73 \\
\hline Rasi2015 & 65.15 & 256.43 & 127.10 & 135.66 & 97.66 & 99.33 & 11.88 & 101.66 & 4.21 & 28.03 & 13.73 & 23.86 & 85.56 & 30.16 & 10.20 \\
\hline BLH116 & 82.27 & 276.43 & 112.80 & 134.66 & 95.33 & 98.33 & 15.80 & 115.66 & 4.51 & 28.93 & 16.33 & 26.58 & 99.70 & 30.10 & 16.23 \\
\hline MM2033 & 83.06 & 286.40 & 137.70 & 135.00 & 97.66 & 99.00 & 14.97 & 149.33 & 4.23 & 28.10 & 14.13 & 24.81 & 97.66 & 32.86 & 17.93 \\
\hline DAS-MH-904 & 87.71 & 234.26 & 86.63 & 137.00 & 96.33 & 100.33 & 12.73 & 116.33 & 4.12 & 25.46 & 14.66 & 25.48 & 87.16 & 29.33 & 11.93 \\
\hline
\end{tabular}




\section{Int.J.Curr.Microbiol.App.Sci (2020) 9(12): 3416-3423}

\begin{tabular}{|c|c|c|c|c|c|c|c|c|c|c|c|c|c|c|c|}
\hline PM16203L & 82.50 & 257.13 & 103.50 & 133.66 & 94.00 & 97.66 & 17.39 & 139.33 & 4.48 & 27.76 & 15.60 & 22.81 & 84.33 & 29.93 & 13.32 \\
\hline Super3366 & 84.58 & 257.13 & 95.63 & 138.00 & 95.00 & 98.66 & 16.40 & 109.00 & 4.39 & 27.46 & 15.33 & 24.02 & 91.20 & 31.50 & 11.56 \\
\hline HT16052 & 81.15 & 258.33 & 104.20 & 134.33 & 98.33 & 102.33 & 11.12 & 106.00 & 3.96 & 26.13 & 14.00 & 20.87 & 89.60 & 31.50 & 9.16 \\
\hline P3522(C) & 74.13 & 293.80 & 131.10 & 133.33 & 95.33 & 98.00 & 13.56 & 165.33 & 4.21 & 28.30 & 14.53 & 26.34 & 89.86 & 32.63 & 16.96 \\
\hline Seedtech2324(C) & 87.84 & 250.83 & 126.56 & 134.33 & 91.33 & 96.66 & 13.23 & 162.00 & 4.46 & 29.80 & 13.93 & 29.86 & 89.45 & 28.03 & 17.00 \\
\hline Buland(C) & 74.28 & 242.96 & 92.20 & 137.00 & 98.33 & 102.00 & 8.12 & 63.33 & 4.87 & 29.00 & 16.20 & 25.32 & 104.83 & 28.03 & 9.10 \\
\hline Bio9681(C) & 83.10 & 281.00 & 114.33 & 132.66 & 92.00 & 95.66 & 11.30 & 121.33 & 4.51 & 27.26 & 17.46 & 25.77 & 93.50 & 31.96 & 14.20 \\
\hline СМн9999 & 84.23 & 239.36 & 86.33 & 137.33 & 92.66 & 96.66 & 19.12 & 160.00 & 4.29 & 28.50 & 13.93 & 31.56 & 83.30 & 28.56 & 19.18 \\
\hline AH8181 & 82.08 & 243.26 & 91.66 & 134.66 & 95.00 & 99.33 & 11.53 & 114.33 & 3.89 & 28.60 & 13.53 & 29.41 & 81.80 & 28.00 & 11.44 \\
\hline MMH17-22 & 81.91 & 261.40 & 135.16 & 135.33 & 96.66 & 99.66 & 8.46 & 124.66 & 4.29 & 27.53 & 16.00 & 24.94 & 85.30 & 30.23 & 13.22 \\
\hline DH291 & 83.98 & 204.20 & 72.60 & 135.00 & 95.33 & 98.00 & 11.57 & 118.00 & 4.25 & 27.23 & 12.46 & 34.42 & 76.53 & 28.40 & 13.26 \\
\hline BLH111 & 83.82 & 267.96 & 100.40 & 137.33 & 95.00 & 98.66 & 12.51 & 133.66 & 4.49 & 29.80 & 13.86 & 33.81 & 83.20 & 33.53 & 18.69 \\
\hline $100 \mathrm{~K}-18$ & 79.93 & 248.76 & 95.40 & 139.00 & 95.66 & 100.00 & 16.45 & 162.66 & 4.37 & 29.30 & 14.73 & 25.21 & 88.23 & 33.00 & 18.55 \\
\hline Bio9637(C) & 84.55 & 227.10 & 98.96 & 135.33 & 94.33 & 99.33 & 11.26 & 174.33 & 3.94 & 27.03 & 13.80 & 22.79 & 66.96 & 34.33 & 17.96 \\
\hline Bio9544(C) & 86.01 & 238.50 & 101.86 & 135.00 & 96.66 & 101.00 & 11.81 & 146.00 & 4.16 & 28.93 & 12.80 & 27.82 & 77.70 & 35.96 & 20.47 \\
\hline DHM117(C) & 72.93 & 231.90 & 92.66 & 135.00 & 101.33 & 102.66 & 11.10 & 106.66 & 4.34 & 29.10 & 13.66 & 26.32 & 92.76 & 29.36 & 9.57 \\
\hline
\end{tabular}

Table.2 Analysis of variance for fifteen quantitative characters in rabi maize

\begin{tabular}{|c|c|c|c|c|c|c|c|c|c|c|c|c|c|c|c|c|}
\hline \multirow[b]{2}{*}{ 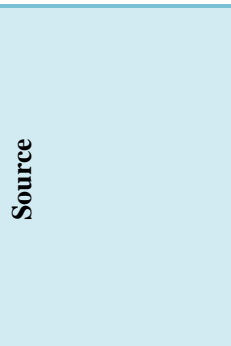 } & \multirow[b]{2}{*}{ 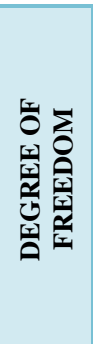 } & \multicolumn{15}{|c|}{ Mean sum of square } \\
\hline & & 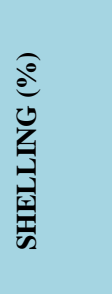 & 它害 & 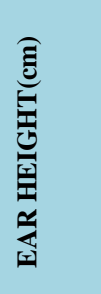 & 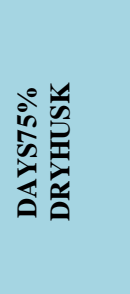 & 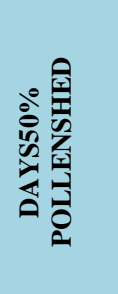 & $\begin{array}{l}0 \\
z \\
1 \\
\overline{1} \\
0 \\
0 \\
0 \\
0 \\
0 \\
0 \\
0\end{array}$ & 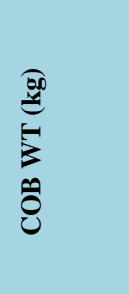 & 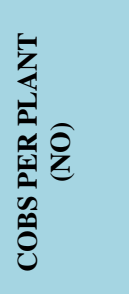 & 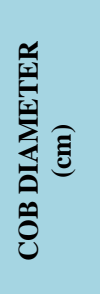 & 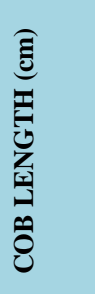 & 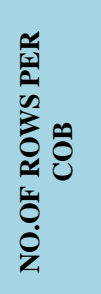 & 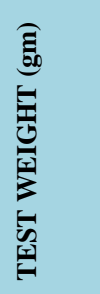 & 氞 & 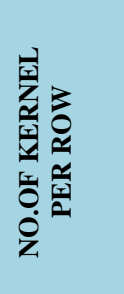 & 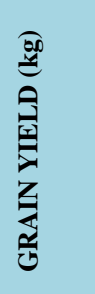 \\
\hline REPLICATION & 2 & 36.03 & 171.57 & 328.49 & 1.300 & 9.43 & 4.90 & 0.117 & 147.10 & $0.091 *$ & $8.97 *$ & 1.16 & 11.17 & 297.71 & $38.59 * *$ & 26.83 \\
\hline GENOTYPES & 39 & $57.16^{*}$ & $1748.93 * * *$ & $\begin{array}{c}969.62 \\
* * *\end{array}$ & $13.48 * * *$ & $16.67 *$ & $6.81 * * *$ & $27.54 * * *$ & $\begin{array}{c}1531.6^{* *} \\
*\end{array}$ & $\begin{array}{c}0.122 * \\
* *\end{array}$ & $\begin{array}{c}7.98 * * \\
*\end{array}$ & $\begin{array}{c}4.34 * * \\
*\end{array}$ & $\begin{array}{c}28.64 * \\
* *\end{array}$ & $\begin{array}{c}213.91 * * \\
*\end{array}$ & $24.24 * * *$ & $27.66^{*}$ \\
\hline ERROR & 78 & 36.25 & 415.64 & 280.23 & 5.16 & 10.72 & 2.96 & 1.34 & 607.03 & 0.027 & 2.82 & 0.41 & 7.37 & 75.03 & 6 & 17.37 \\
\hline
\end{tabular}


Table.3 Phenotypic and genotypic coefficients of variability, heritability and genetic advance for fifteen quantitative characters in rabi maize

\begin{tabular}{|c|c|c|c|c|c|c|c|c|}
\hline & \multirow{2}{*}{$\begin{array}{c}\text { Grand } \\
\text { mean }\end{array}$} & \multicolumn{2}{|c|}{ Range } & \multicolumn{2}{|c|}{$\begin{array}{l}\text { Coefficient of } \\
\text { Variation }(\%)\end{array}$} & \multirow{2}{*}{$\begin{array}{l}\text { Heritab } \\
\text { ility } \\
(\%)\end{array}$} & \multirow[t]{2}{*}{$\begin{array}{l}\text { Genetic } \\
\text { advance }\end{array}$} & \multirow{2}{*}{$\begin{array}{l}\text { Genetic advance } \\
\text { as percent of } \\
\text { mean }\end{array}$} \\
\hline & & Min & Max & GCV & PCV & & & \\
\hline SHELLING (\%) & 82.65 & 65.15 & 87.84 & 3.19 & 7.95 & 16.1 & 2.183 & 2.64 \\
\hline PLANT HEIGHT(cm) & 262.33 & 204.200 & 304.50 & 8.03 & 11.17 & 51.7 & 31.21 & 11.90 \\
\hline EAR HEIGHT(cm) & 111.71 & 72.60 & 145.00 & 13.57 & 20.21 & 45.1 & 20.96 & 18.76 \\
\hline DAYS $75 \%$ DRY HUSK & 135.82 & 132.33 & 142.33 & 1.22 & 2.07 & 35.0 & 2.02 & 1.49 \\
\hline $\begin{array}{c}\text { DAYS 50\% POLLEN } \\
\text { SHED }\end{array}$ & 95.64 & 91.33 & 101.66 & 1.47 & 3.72 & 15.6 & 1.146 & 1.19 \\
\hline DAYS 50\% SILKING & 99.20 & 95.66 & 102.66 & 1.14 & 2.07 & 30.2 & 1.28 & 1.29 \\
\hline COB WT (kg) & 14.56 & 8.12 & 19.90 & 20.29 & 21.80 & 86.6 & 5.66 & 38.90 \\
\hline $\begin{array}{c}\text { COBS PER PLANT } \\
\text { (NO) }\end{array}$ & 126.17 & 63.33 & 174.33 & 13.91 & 23.97 & 33.7 & 20.98 & 16.63 \\
\hline COB DIAMETER $(\mathrm{cm})$ & 4.32 & 3.89 & 4.87 & 4.09 & 5.62 & 53.1 & 0.266 & 6.14 \\
\hline COB LENGTH (cm) & 27.22 & 24.00 & 29.80 & 4.81 & 7.83 & 37.8 & 1.66 & 6.10 \\
\hline $\begin{array}{c}\text { NO.OF ROWS PER } \\
\text { COB }\end{array}$ & 14.67 & 12.46 & 17.46 & 7.80 & 8.94 & 76.2 & 2.06 & 14.03 \\
\hline TEST WEIGHT (gm) & 27.01 & 20.87 & 34.42 & 9.85 & 14.08 & 49.0 & 3.84 & 14.22 \\
\hline $\begin{array}{c}\text { EAR LEAF LENGTH } \\
(\mathrm{cm})\end{array}$ & 88.64 & 66.96 & 104.83 & 7.67 & 12.42 & 38.2 & 8.65 & 9.76 \\
\hline $\begin{array}{l}\text { NO.OF KERNEL PER } \\
\text { ROW }\end{array}$ & 31.02 & 26.20 & 39.06 & 7.94 & 11.20 & 50.3 & 3.60 & 11.61 \\
\hline GRAIN YIELD (kg) & 14.47 & 9.10 & 20.47 & 13.15 & 32.37 & 16.5 & 1.55 & 11.00 \\
\hline
\end{tabular}




\section{Genetic advance}

The higher estimate of genetic advance as percent of mean was exhibited by cob wt $(38.90 \%)$, whereas moderate estimate of genetic advance as percent of mean were exhibited by ear height $(18.76 \%)$, cobs per plant $(16.63 \%)$, test weight $(14.22 \%)$, no. of rows per cob(14.03\%), plant height(11.90\%), no. of kernel per row $(11.61 \%)$, grain yield (11.0\%), Lower estimates of genetic advance as percent of mean were shown by ear leaf length $(9.76 \%)$, cob diameter $(6.14 \%)$, cob length $(6.10 \%)$, shelling $(2.64 \% \%)$, days $75 \%$ dry husk (1.49\%), days 50\% silking (1.29\%), days $50 \%$ pollen shed $(1.19 \%)$. These results are in agreement with the findings of Shakoor et al.,(2007), Kumar et al., (2006), Kumar, and Satyanarayana (2001), Singh et al., (2006)Sesay et al., (2016), and Nataraj et al.,(2014).

According to Johnson et al., (1955) heritability used in conjunction with genetic advance provides better information for selecting the best individuals then the heritability alone. High to moderate estimates of heritability accompanied with high to moderate genetic advance for indicated the predominance of additive gene action for the expression of the characters. Hence selection of the characters with moderate to high estimates of heritability accompanied with high to moderate genetic advance would be effective in this population.

A perusal of the Table 3, where in the results of PCV, GCV, heritability and genetic advance have been furnished, revealed that selection for cob wt, grain yield, ear length, no of cobs per plant, test weight, ear height would be effective for improvement of yield in this population.

In conclusion the analysis of variance revealed significant differences among all the characters under study in the forty maize genotypes. Thus the findings show that, sufficient amount of variability were present in this maize population. High GCV and PCV were observed for cob wt., and moderate values for cobs per plant, ear height, and grain yield emphasizing the existence of variation suggesting simple selection for these traits would be effective. High heritability with high genetic advance was recorded in cob weight, and no. of rows per cob while moderate heritability and genetic advance was recorded for plant height, ear height, no. of kernel per row, test weight, no. of rows per cob, cobs per plant, ear leaf length, cob length, and grain yield indicating greater importance for additive gene effect, and possibility of improvement of these traits through simple selection, mass selection and pedigree method of plant breeding might be effective.

\section{References}

Akbar, M., Shakoor, M.S., Hussain, A. and Sarwar, M. (2008). 'Evaluation of maize 3 way crosses through genetic variability, broad sense heritability, character association and path analysis.' J. Agric. Res. Lahore., 46(1), pp. 39-45.

Begum, S., Ahemed, A., Omy, S.H., Rohmann, M.M and Amriuzzaman, M. (2016). 'Genetic variability, character association and path analysis in maize (Zea mays L.).' Bangladesh Journal of Agricultural Research, 41(1), pp. 173182.

Bekele, A and Rao, T. N. (2014). 'Estimates of Heritability, Genetic Advance and Correlation Study for Yield and It's Attributes in Maize (Zea mays L.)', Journal of Plant Sciences, 2(1), pp. 1-4. doi: 10.11648/j.jps.20140201.11.

Ghosh, A., Subba, V., Roy, A., Ghosh, A., and Kundagrami, S. (2014) 'Genetic variability and character association of 
grain yield components in some inbred lines of maize (Zea mays L.)', Journal of Agroecology and Natural Resource Management, 1(2), pp. 34-39.

Hanson, G.H., Robinson, H.F. and Comstock, R. E. (1956). 'Biometrical studies of yield in segregating populations of Korean hespedeza', Agron. J., 48, pp. 267-282.

Johnson, H. W., Robinson, H.F and Comstock, R. E. (1955). 'Estimates of genetic and environmental variability in soybean', Agronomy journal, 47, pp. 314-318.

Kumar, S., J.P., Singh, J and Singh, S. P. (2006). 'Correlation and path analysis in early generation inbreds of maize (Zea mays L.)', Crop Improvement., 33(2), pp. 156-160.

Nataraj, V., Shahi J.P and Vandana, D. (2014). 'Estimation of variability, heritability and genetic in certain inbreds of maize (Zea mays L.)', International journal of Applied Biology and Pharmaceutical Technology, 5(1), pp. 205-208.

Ojo, D.K., Omikunle, O.A., Oduwaye, O.A., Ajala, M.O and Ogunbayo, S.. (2006) 'Heritability, character correlation and path coefficient analysis among six inbread lines of maize (Zea mays L.)', World Journal of Agricultural Science, 2(3), pp. 352-358.

Panse, V.G., S. (1957) 'Genetics of competitive characters in relation to plant breeding', Indian Journal of Genetics and Plant Breeding, 17, pp. 318-326.

Pradeep Kumar, P. and Satyanarayana, E.
(2001) 'Variability and correlation studies of fall season inbred lines of maize.' J. Res., ANGRAU, Hyderabad, India, 29: pp. 71-75.

Robinson, H.F., Cornstock, R.E. and Harvey, P. H. (1949). 'Estimates of heritability and degree of dominance in corn.', Agron. J., 41, pp. 353-359.

Sesay, S. et al., (2016). 'Genetic variability, heritability and genetic advance studies in top-cross and three-way cross maize (Zea mays L) hybrids', Maydica.

Shakoor, M. S., Akbar, M. and Hussain, A. (2007). 'Correlation and path coefficients studies of some morphophysiological traits in maize double crosses', Pakistan Journal of Agricultural Sciences, 44(2), pp. 213216.

Sharma, R., Maloo, S. R., and Joshi, A. (2014). 'Research Note Genetic variability analysis in diverse maize genotypes (Zea mays L.).', Electronic Journal of Plant Breeding, 5(3), pp. 545-551.

Singhal, N., Verma, S.S., Baskheti, D.C and Kumar, A. (2006). 'Heritability, genetic advance, correlation and path coefficient estimation in high quality protein maize (Zea mays L.).' Asian journal of Biosciences, 1(2), pp. 54-56.

Sumathi, P., Nirmalakumari, A. and Mohanraj, K. (2005). 'Genetic variability and traits interrelationship studies in industrially utilized oil rich CIMMYT lines of maize (Zea mays L)', Madras Agric. J, 92(10-12), pp. 612617.

\section{How to cite this article:}

Abhishek Sadhu, Amitava Paul, P. C. Kole and Sandip Debnath. 2020. Genetic Variability, Heritability and Genetic Advance Studies on Rabi Maize (Zea mays L.). Int.J.Curr.Microbiol.App.Sci. 9(12): 3416-3423. doi: https://doi.org/10.20546/ijcmas.2020.912.406 